\title{
Optical Coherence Tomography in Diagnosis of Primary Open Angle Glaucoma
}

\author{
Suzana Nuellari ${ }^{1}$, Julinda Jaho $^{2}$ \\ ${ }^{1}$ Polyclinic of Specialities No 3, Tirana, Albania \\ ${ }^{2}$ University Hospital Centre "Mother Theresa" Tirana, Albania
}

\begin{abstract}
Glaucoma is a group of eye diseases traditionally characterized by elevated intraocular pressure (IOP). However, glaucoma is more accurately defined as an optic neuropathy than a disease of high pressure.The aim of the study was to asses retinal nerve fiber layer (RNFL) thickness in glaucomatous eyes using spectral domain optical coherence tomography (SD-OCT) and to study their correlations with glaucomatous damage. This cohort observational study was carried out at outpatients clinic in city of Tirana during the period 2013 -2014. A total of 82 patients were enrolled in our study so fulfilling the criteria of preliminary glaucoma suspect, with Optic $C: D$ ratio of more than 0.6 at least in one eye, intra ocular pressure (IOP) higher than $20 \mathrm{~mm} \mathrm{Hg}$, and age more than 20 years. Patients had a mean age of $55 \pm 12$ years. Out of 82 patients, 31 (38\%) patients were female and 51 (62\%) were male. Mean IOP was $25 \pm 5$. Out of these 82 suspects, 24 (29\%) patients were found to have nerve fibre layer thickness outside normal limits i.e. decreased in half a quadrant or more. Significantly lower values of the mean pRNFL thickness were found in the POAG (62.10 $\pm 14.45 \mu m)$ and groups as compared with GS group (100.0 $\pm 7.15 \mu m), p<0.01$.Glaucoma can be easily screened by routine ophthalmoscopy done by general medical practitioner.
\end{abstract}

Keywords: glaucoma, intraocular pressure, retinal nerve, fiber layer

\section{Introduction}

Glaucoma is a group of eye diseases traditionally characterized by elevated intraocular pressure (IOP). However, glaucoma is more accurately defined as an optic neuropathy than a disease of high pressure. In open-angle glaucoma, optic nerve damage results in a progressive loss of retinal ganglion cell axons, which is manifested initially as visual field loss and, ultimately, irreversible blindness if left untreated (1). This topic will discuss the epidemiology, clinical presentation, and diagnosis of open-angle glaucoma in adults. Glaucoma in children, angle-closure glaucoma, and treatment and prevention of open-angle glaucoma are discussed elsewhere. There are different types of glaucoma, generally categorized by the anterior chamber (iridocorneal) angle and the underlying etiology, if known. Open-angle glaucoma is an optic neuropathy characterized by progressive peripheral visual field loss followed by central field loss, in a characteristic pattern. This is usually but not always in the presence of elevated intraocular pressure (IOP), perhaps in part related to increased aqueous production and decreased outflow. The optic nerve or "disc" takes on a hollowed-out appearance on ophthalmoscopic examination, which is described as "cupping." Cupping is associated with the loss of ganglion cell axons (2). Angleclosure glaucoma is characterized by narrowing or closure of the anterior chamber angle. The normal anterior chamber angle provides drainage for the aqueous humor (the fluid that fills the eyeball). When this drainage pathway is narrowed or closed, inadequate drainage leads to elevated intraocular pressure and damage to the optic nerve. Acute angle-closure glaucoma occurs in eyes with a certain anatomical predisposition. It presents as a painful red eye and must be treated within 24 hours to prevent permanent blindness. The detection of early glaucomatous damage is one of the most important aspects of glaucoma management. Newer versions of imaging devices for the optic nerve head $(\mathrm{ONH})$ and retinal nerve fiber layer (RNFL) are introduced with the expectation that higher resolution would lead to more reproducible measurements, better clinical performance and diagnosis of preprerimetric glaucoma $(3,4)$. As glaucomatous structural damage is widely accepted to precede functional damage $(5,6)$. Hence currently available imaging techniques used for detection of glaucoma including confocal scanning laser ophthalmoscopy (CSLO), optical coherence tomography (OCT), and scanning laser polarimetry (SLP) have gained much clinical interest. It is uncertain whether the RNFL or the neuroretinal rim represents a more sensitive surrogate for detecting glaucomatous change (7). Moreover, since these techniques use different methods to measure different aspects of the eye, it is possible to expect that their measurements have different associations with glaucoma diagnosis. The aim of the study was to asses retinal nerve fiber layer (RNFL) thickness in glaucomatous eyes using spectral domain optical coherence tomography (SD-OCT) and to study their correlations with glaucomatous damage.

\section{Material and Methods}

This cohort observational study was carried out at outpatients clinic in city of Tirana during the period 2013 2014. A total of 82 patients were enrolled in our study so fulfilling the criteria of preliminary glaucoma suspect, with Optic C:D ratio of more than 0.6 at least in one eye, intra ocular pressure (IOP) higher than $20 \mathrm{~mm} \mathrm{Hg}$, and age more than 20 years. Subjects previously diagnosed as cases of glaucoma (POAG, PACG and secondary glaucomas), previous intra ocular surgery, and optic neuropathy due to other causes were excluded. Systemic diseases were also ruled out .Permission was taken from hospital ethical committee. Written informed consent was taken. Both IOP and OCT images were taken on the same day with calibrated instruments. Intraocular pressure of both eyes was measured with help of Goldmannapplanation tonometer using $2 \%$ fluorescein eye drops by the same physician to avoid inter

\section{Volume 4 Issue 12, December 2015}




\section{International Journal of Science and Research (IJSR) \\ ISSN (Online): 2319-7064}

Index Copernicus Value (2013): 6.14 | Impact Factor (2014): 5.611

examiner and inter tonometer variation, between 9 to $11 \mathrm{AM}$ to minimize the effect of diurnal variation. Central corneal thickness was also measured. Three readings of each eye were taken at 30 minutes interval and mean calculated. OCT images were taken using Heidelberg HRA+OCT Spectralis by a single person to avoid inter examiner error. Assessment of peripapillary RNFL thickness was made with circular spectral domain optical coherence tomography (SD-OCT) scans. RNFL measurements in each patient's eye were obtained in temporal, superior, nasal, and inferior quadrants. Analysis of variance (ANOVA) was used for comparison of RNFL parameters among various study groups. The relationship of RNFL parameters with visual field (VF) global indices was evaluated with regression analysis and the coefficient of determination $\left(\mathrm{r}^{2}\right)$ was calculated.

\section{Results and Discussion}

Patients had a mean age of $55 \pm 12$ years. Out of 82 patients, $31(38 \%)$ patients were female and $51(62 \%)$ were male. Mean IOP was $25 \pm 5$. Out of these 82 suspects, 24 (29\%) patients were found to have nerve fibre layer thickness outside normal limits i.e. decreased in half a quadrant or more (fig. 1) They were further investigated and documented with visual field analysis and IOP phasing.Significantly lower values of the mean pRNFL thickness were found in the POAG $(62.10 \pm 14.45 \mu \mathrm{m})$ and groups as compared with GS group $(100.0 \pm 7.15 \mu \mathrm{m}), \mathrm{p}<0.01$ (fig.2). Also, in pairwise group comparison RNFL in glaucomatous eyes was significantly thinner in all quadrants $\mathrm{p}<0.05$. The mean deviation (MD) was significantly correlated with pRNFL thickness in POAG group $\left(r^{2}=0.27\right.$ $\mathrm{p}<0.01$ ). Glaucoma is a progressive disease characterized by death of ganglion cells and degeneration of retinal nerve fiber layer leading to irreversible loss of vision $(8,9)$.Although automated perimetry has been the standard method for detecting progressive disease, it is known that many patients can have progressive structural damage that precedes detectable associated changes in the visual field. There are three main parameters relevant to the detection of glaucomatous loss i.e. retinal nerve fiber layer, optic nerve head, and the "ganglion cell complex." Latest spectral domain ocular coherence tomography (SD-OCT) was used to measure retinal nerve fiber layer (RNFL) thickness around optic nerve head. SD-OCT can directly measure and quantify RNFL thickness by calculating the area between the internal limiting membrane (ILM) and RNFL border (how the edge of the RNFL is determined and how blood vessels are handled is different between different machines, which do not have interchangeable measurement outputs) (10). Its software was used to see any abnormality in retinal nerve fiber layer thickness in different quadrants around optic nerve by comparing with normative preloaded data in the software. SD-OCT is a superior technology than conventional time domain (TD- OCT) with reference to scanning speed up to 200 times faster and higher axial resolution (3 to $6 \mu \mathrm{m}$ ). Progressive RNFL thinning measured on SD-OCT can often be used to detect progressive disease (11). The top three RNFL progression patterns are: widening of an existing RNFL defect, deepening without widening of an existing RNFL defect, or development of a new RNFL defect. In one study, the inferotemporal quadrant was the most frequent location for RNFL progression (12). In such pre perimetric disease, SD-OCT RNFL is especially useful in helping to diagnose glaucoma prior to the onset of visual field loss. In the presence of perimetric disease, finding RNFL bundle loss on SD-OCT with a corresponding abnormality in the visual field served by those retinal ganglion cells can help confirm the diagnosis of glaucoma (13). In early to moderate glaucoma, progressive thinning of RNFL thickness measured by SD-OCT is a very useful tool to judge progression of disease. At advanced stages however, SD-OCT is less clinically useful due to a "floor effect" of RNFL thickness. With advanced loss, RNFL thickness levels off, rarely falling below $50 \mu \mathrm{m}$ and almost never below $40 \mu \mathrm{m}$ due to the assumed presence of residual glial or non-neural tissue including blood vessels (14). At this level of disease, serial visual fields are more useful to judge progression.

\section{Conclusion}

Glaucoma can be easily screened by routine ophthalmoscopy done by general medical practitioner. Any glaucoma suspect can be diagnosed early by using new imaging technique like ocular coherence tomography nerve fiber layer thickness analysis by an ophthalmologist. Doubtful cases of glaucoma should be further investigated and documented with visual field analysis and IOP phasing. SD-OCT is a powerful objective structured assessment tool that can greatly assist clinicians in diagnosing and managing glaucoma (especially early disease), when used in conjunction with visual field testing and clinical examinations.

\section{References}

[1] Kong X, Chen Y, Chen X, Sun X. Influence of family history as a risk factoron primary angle closure and primary open angle glaucoma in a Chinesepopulation. Ophthalmic Epidemiol. 2011;18:226-32.

[2] Coleman AL, Miglior S. Risk factors for glaucoma onset and progression.SurvOphthalmol. 2008;11;53:S3 10 .

[3] Skaf M, Bernardes AB, Cardillo JA, et al. Retinal nerve fibre layer thickness profile in normal eyes using third generation OCT. Eye. Published online July 25, 2005.

[4] Bowd C, Zangwill LM, Berry CC, et al. Detecting early glaucoma by assessment of retinal nerve fiber layer thickness and visual function. Invest Ophthalmol Vis Sci. 2001; 42: 1993-2003.

[5] Kanamori A, Nagai-Kusuhara A, Escano MF, Maeda H, Nakamura M, Negi A. Comparison of confocal scanning laser tomography, scanning laser polarimetry and optical coherence tomography to discriminate ocular hypertension and glaucoma at an early stage.

[6] Mayoral F, Polo V, Ferreras A, Larrosa JM, Pueyo V, Honrubia F. Diagnostic ability of stratus optical Coherence tomography (OCT) in pre-perimetric Glaucoma diagnosis. Arch Soc ESP Ophthalmol 2006; 81: 537-544.

[7] Ake Tzu-Hui Lu, Mingwu Wang, Rohit Varma et al, Combining Nerve Fibre Layer to Optimize Glaucoma Diagnosis with Optical Coherence Tomography, NIHPA Author Manuscripts, Ophthalmology. Aug 2008; 115(8): 1352-1357.e2.

\section{Volume 4 Issue 12, December 2015}




\section{International Journal of Science and Research (IJSR) \\ ISSN (Online): 2319-7064}

Index Copernicus Value (2013): 6.14 | Impact Factor (2014): 5.611

[8] Caprioli J, Mahadavi KN, Law SK, Badala F. Optic disc imaging in perimetrically normal eyes of Glaucoma patients with unilateral field loss. Trans Am Ophthalmolsoc. 2006; 104: 202-211.

[9] Rosa MG, Hernandez MG, Lopez VL, Mendez MS, Vega RR. Optic disc tomography and perimetry in controls, Glaucoma Suspects and early and established Glaucomas. American Academy of Optometry 2007; 84: 33-41

[10] Schuman JS, Hee MR, Puliafito CA, et al. Quantification of nerve fiber layer thickness in normal and glaucomatous eyes using optical coherence tomography. Arch Ophthalmol. 1995; 113: 586-596

[11] Budenz DL, Michael A, Chang RT, McSoley J, Katz J. Sensitivity and specificity of the Stratus OCT for perimetric glaucoma. Ophthalmology. 2005; 112: 3-9.

[12] Kanamori A, Nakamura M, Escano M F et al, Evaluation of the glaucomatous damage on retinal nerve fibre layer thickness measured by optical coherence tomography. Am J Ophthalmol. 2003 Apr; 135(4): 51320.

[13] SergiosTaliantzisPapaconstantinom D, Koutsandreac et al, Comparative studies of RNFL thickness measured by OCT with global index of visual fields in patients with ocular hypertension and early open angle glaucoma. ClinOphthalmol. 2009; 3: 373-379.

[14] Yalvac, AltunsoyM,Cansever S et al, The correlation between visual field defects and focal nerve fibre layer thickness measured with optical coherence tomography in the evaluation of glaucoma. J Ophthalmology 2009 Jan; 18(1): 53-61.

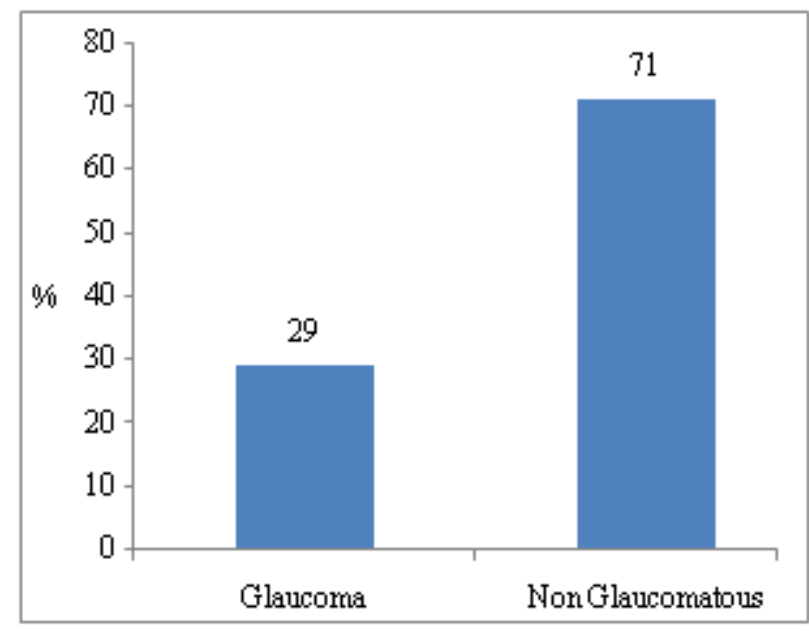

Figure 1: Glaucoma suspected patients $(n=82)$

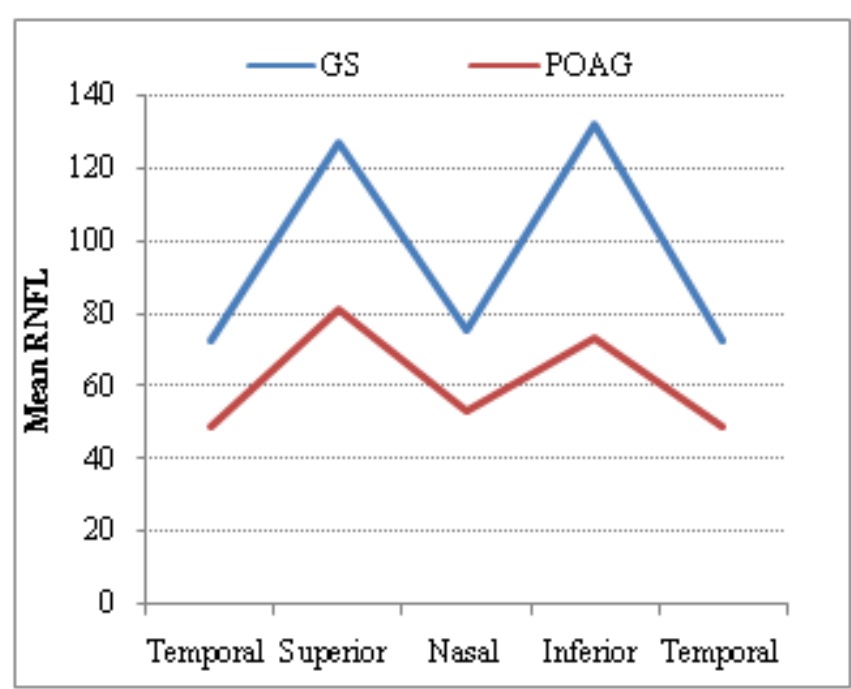

Figure 2: Retinal nerve fiber layer thickness (micrometers) in each quadrant for 2 study groups 